Introduction In many chronic diseases vitamin $\mathrm{D}$ has been proposed as an adjunctive anti-inflammatory therapy. Vitamin D upregulates MKP1, thereby downregulating p38 phosphorylation and the NFKB inflammatory cascade (Zhang et al, J Immunol. 2012;188(5):2127-35). Steroids exert anti-inflammatory effects via this cascade, and exhibit synergy with vitamin $\mathrm{D}$ for some effects (Yu et al, Journal of the National Cancer Institute. 1998;90(2):134-41). Patients with COPD have chronic pulmonary inflammation, with upregulation of NFKB, yet do not exhibit a good response to steroids. Vitamin D therapy has been trialled in COPD patients, albeit with disappointing results (Lehouck et al, Annals of internal medicine. 2012;156(2):10514). We hypothesised that COPD patients' inflammatory response would differ from health, and that vitamin D would exhibit synergy with steroids in vitro to improve this.

Methods PBMCs isolated from 10 COPD patients and 10 healthy control subjects were incubated with LPS, vitamin D, dexamethasone, a p38 MAPK inhibitor or combinations of these agents. Supernatants were harvested for TNF and IL6 measurements (ELISA).

Results LPS caused a marked rise in IL6 in both healthy controls $(p=0.044)$ and COPD patients $(p=0.008)$. IL6 reduction with vitamin $\mathrm{D}$ was only seen in health. IL6 reduction with addition of dexamethasone was not statistically significant $(\mathrm{p}=$ 0.636) in COPD. Combinations of agents failed to produce any additional benefit in both health and COPD.

The response to vitamin $\mathrm{D}$ was heterogeneous; half of healthy subjects showed an anti-inflammatory response but in COPD only $12.5 \%$ of patients exhibited this. The difference in response rate was not significant $(\mathrm{p}=0.120$, Fishers exact test), though this may be due to low power. Similarly reduced response rate to dexamethasone was seen in COPD.

Conclusion Vitamin D does not enhance the anti-inflammatory effect of steroids. The anti-inflammatory effects of vitamin D are no different between COPD and health; variability of response may be one reason for lack of effect of vitamin D in clinical trials to date in COPD patients.

\section{M138 DO STANDARD CARDIOVASCULAR RISK SCORES IDENTIFY RISK IN PATIENTS WITH COPD?}

ME John, S Hussain, M Al Haddad, CE Bolton. University of Nottingham, Nottingham, UK

\subsection{6/thoraxjnl-2014-206260.433}

Introduction Patients with COPD have increased risk of cardiovascular (CV) disease compared to smokers without COPD, ${ }^{1}$ with over $25 \%$ of deaths CV related. $^{2}$ Several CV risk calculators for the general population exist but it is unclear whether they are applicable for COPD.

Hypothesis

Standard CV risk calculators do not identify the increased risk in patients with COPD.

Methods Subjects with a smoking history $>10$ pack years, with and without COPD, were assessed at clinical stability, COPD n $=191$ and controls $\mathrm{n}=106$. Post-bronchodilator spirometry and blood pressure were performed, blood taken for lipids and self-reported medical and smoking history recorded. In those without documented established CV disease or diabetes (COPD $\mathrm{n}=135$ and controls $\mathrm{n}=88$ ), 10 year CV risk was calculated using ACC/ $\mathrm{AHA}^{3}$ and NHLBI[4] calculators.

Results Both groups were well matched for gender and mean arterial blood pressure (MAP), with the COPD group slightly older, Table 1. Mean CV risk scores were similar between

\begin{tabular}{lll} 
Abstract M138 Table $\mathbf{1}$ & \\
\hline $\begin{array}{l}\text { Mean (SD) } \\
\text { unless stated otherwise }\end{array}$ & COPD & Controls \\
\hline $\mathrm{n}$ & 135 & 88 \\
Age (years) & $67(8)$ & $65(10)$ \\
Gender male $\mathrm{n}(\%)$ & $79(59)$ & $56(64)$ \\
FEV $\%$ predicted & $56(19)$ & $100(14)$ \\
Smoking pack years & $45(26)$ & $29(19)$ \\
Smoking status current: ex $\mathrm{n}(\%)$ & $40(30): 95(70)$ & $18(20): 70(80)$ \\
MAP (mmHg) & $105(13)$ & $105(12)$ \\
ACC/ AHA risk score & $19(12)$ & $16(12)$ \\
NHLBI risk score & $11(8)$ & $10(8)$ \\
\hline
\end{tabular}

patients with COPD and controls, Table 1, ACC/AHA p $=0.16$ and NHLBI $\mathrm{p}=0.59$. When using an established cut-off point of $20 \%$ for high 10 year CV risk, similar proportions were identified as high risk: the ACC/AHA calculator - $37 \%$ of the patients with COPD and 33\% of controls; and with the NHLBI calculator $15 \%$ of the patients with COPD and $10 \%$ of controls were identified as high-risk.

Discussion Although nearly double the proportion of patients with COPD compared to controls with a smoking history have current $\mathrm{CV}$ disease or diabetes in this cross-sectional study, the increased risk of future incident $\mathrm{CV}$ disease in patients with COPD was not identified using standard calculators.

Supported by a NIHR BRF Fellowship.

\section{REFERENCES}

1 Feary, JR, et al. Thorax, 2010;65(11): p. 956-62

2 McGarvey, LP, et al. Thorax, 2007;62(5): p. 411-5

3 ACC/AHA Available from: http://my.americanheart.org/professional/StatementsGuidelines/Prevention-Guidelines_UCM_457698_SubHomePage.jsp

4 NHLBI Available from: http://crdrisk.nhlbi.nih.gov/

\section{M139 FRAILTY AND PREMATURE CARDIOVASCULAR AGEING IN COPD}

AM Albarrati, NS Gale, S Enright, M Munnery, I Munnery, S Saikia, JR Cockcroft, DJ Shale. Cardiorespiratory Medicine, Wales Heart Institute, Cardiff University, Cardiff, UK

\subsection{6/thoraxjn|-2014-206260.434}

Background Presence of comorbidities in chronic obstructive pulmonary disease (COPD) parallels the accumulation of multiple system deficits associated with ageing and assessed as frailty. An important association of frailty in the elderly is increased cardiovascular disease, which is also a major cause of mortality in COPD. ${ }^{1}$ However, frailty has not been extensively studied in COPD. We hypothesised that frailty in COPD would be associated with biomarkers of greater systemic involvement including cardiovascular and indicating premature cardiovascular ageing.

Methods Frailty was determined as a Frailty Index (FI) using the 61-element comprehensive geriatric assessment questionnaire in 500 patients with stable COPD, confirmed with spirometry, and 150 non-COPD comparators. This cross-sectional study was taken from within the ARCADE study. Other assessments included body composition; handgrip strength (HGS); aortic pulse wave velocity (PWV); cardiac haemodynamics; 6 min walk distance (6MWD); Timed Up and Go (TUG) test; St George's Respiratory Questionnaire (SGRQ) and C-reactive protein (CRP). The FI was calculated by dividing the number of deficits that the patient had by the maximum, 61

Results Patients and comparators were similar for age, BMI and gender proportion. The FI was greater in the COPD group; 